\title{
Schauder bases and the decay rate of the heat equation
}

\author{
José BONET, WOLFGANG LUSKY AND JARI TASKINEN
}

Abstract. We consider the classical Cauchy problem for the linear heat equation and integrable initial data in the Euclidean space $\mathbb{R}^{N}$. We show that given a weighted $L^{p}$-space $L_{w}^{p}\left(\mathbb{R}^{N}\right)$ with $1 \leq p<\infty$ and a fast-growing weight $w$, there are Schauder bases $\left(e_{n}\right)_{n=1}^{\infty}$ in $L_{w}^{p}\left(\mathbb{R}^{N}\right)$ with the following property: given a positive integer $m$, there exists $n_{m}>0$ such that, if the initial data $f$ belong to the closed linear space of $e_{n}$ with $n \geq n_{m}$, then the decay rate of the solution of the heat equation is at least $t^{-m}$. Such a basis can be constructed as a perturbation of any given Schauder basis. The proof is based on a construction of a basis of $L_{w}^{p}\left(\mathbb{R}^{N}\right)$, which annihilates an infinite sequence of bounded functionals.

\section{Introduction}

Given an integrable function $f \in L^{1}\left(\mathbb{R}^{N}\right)$ in the Euclidean space $\mathbb{R}^{N}, N \in \mathbb{N}$, the unique solution of the classical Cauchy problem for the linear heat (or diffusion) equation

$$
\begin{aligned}
\partial_{t} u(x, t) & =\Delta u(x, t) \text { for } x \in \mathbb{R}^{N}, \quad t>0 \\
u(x, 0) & =f(x) \text { for } x \in \mathbb{R}^{N},
\end{aligned}
$$

has the decay rate $t^{-N / 2}$ for large "times" $t$ :

$$
\|u(\cdot, t)\|_{\infty}:=\sup _{x \in \mathbb{R}^{N}}|u(x, t)| \leq C t^{-N / 2}
$$

(for simplicity, we will only consider the decay rates measured in the sup-norm of the $x$-space). This follows directly from the solution formula

$$
u(x, t)=\mathrm{e}^{t \Delta} f(x):=\frac{1}{(2 \pi t)^{N / 2}} \int_{\mathbb{R}^{N}} \mathrm{e}^{-\frac{1}{4 t}(x-y)^{2}} f(y) \mathrm{d} y,
$$

where we write $x^{2}:=|x|^{2}=\sum_{j=1}^{N} x_{j}^{2}$ for vectors $x=\left(x_{1}, \ldots, x_{N}\right) \in \mathbb{R}^{N}$ and $\Delta=$ $\sum_{j=1}^{N} \partial_{j}^{2}=\sum_{j=1}^{N}\left(\partial / \partial x_{j}\right)^{2}$ for the Laplacian. For general initial data $f \in L^{1}\left(\mathbb{R}^{N}\right)$, which is not necessarily positive, cancellation phenomena may cause faster decay rates. For example, in the case $N=1$, if $f$ is such that $\int_{-\infty}^{\infty} f(x) \mathrm{d} x=0$, then a simple argument shows that $e^{t \Delta} f$ decays at least with the speed $t^{-1}$. 
To describe our main result on decay rates, we fix a continuous weight function $w: \mathbb{R}^{N} \rightarrow \mathbb{R}^{+}$with symmetry $w(x):=w(-x)$ for all $x \in \mathbb{R}^{N}$. We assume $w$ is fast growing which means that

$$
\sup _{x \in \mathbb{R}^{N}} \frac{1}{w(x)}(1+|x|)^{m}<\infty \quad \forall m \in \mathbb{N} .
$$

Given $p \in[1, \infty)$, we denote by $L_{w}^{p}\left(\mathbb{R}^{N}\right)$ the weighted $L^{p}$-space on $\mathbb{R}^{N}$ endowed with the norm

$$
\|f\|_{p, w}:=\left(\int_{\mathbb{R}^{N}}|f(x)|^{p} w(x) \mathrm{d} x\right)^{1 / p} .
$$

Our main result, in addition to Theorem 2.2 on Schauder bases which annihilate linear functionals, reads as follows (definitions related to Schauder bases are recalled below):

THEOREM 1.1. Let $1 \leq p<\infty$ and let the weight $w$ satisfy the conditions above. There exists a Schauder basis $\left(e_{n}\right)_{n=1}^{\infty}$ of the Banach space $L_{w}^{p}\left(\mathbb{R}^{N}\right)$ with the following property: given $m \in \mathbb{N}$, there exists $n_{m} \in \mathbb{N}$ such that any initial data $f$

$$
f=\sum_{n=1}^{\infty} f_{n} e_{n} \in L_{w}^{p}\left(\mathbb{R}^{N}\right),
$$

with the property $f_{n}=0$ for all $n=1, \ldots, n_{m}$, has the fast decay property

$$
\left\|\mathrm{e}^{t \Delta} f\right\|_{\infty} \leq \frac{C}{t^{m / 2}}\|f\|_{p, w}
$$

for all $t \geq 1$.

Moreover, if $p>1$, given any Schauder basis $\left(\tilde{e}_{n}\right)_{n=1}^{\infty}$ of the space $L_{w}^{p}\left(\mathbb{R}^{N}\right)$ and an arbitrary $0<\varepsilon<1$, one can find a basis $\left(e_{n}\right)_{n=1}^{\infty}$ with the above described property such that the linear map (change of basis operator) defined by $T \tilde{e}_{n}=e_{n}$ is an isomorphism from $L_{w}^{p}\left(\mathbb{R}^{N}\right)$ onto itself satisfying

$$
\|\mathrm{id}-T\|<\varepsilon,
$$

where id is the identity operator on $L_{w}^{p}\left(\mathbb{R}^{N}\right)$.

In other words, if initial data are included in the finite co-dimensional subspace $G_{m}=\overline{\operatorname{sp}\left(e_{n}: n \geq n_{m}\right)}$, then the corresponding solution decays at least at the speed $t^{-m}$; leaving out finitely many coordinates in the Banach space of initial data makes the solution decay fast. The subspace $G_{m}$ thus has an explicit description in terms of the Schauder basis.

The last statement means that such a basis can be obtained as a "perturbation" of any given basis.

REMARK 1.2. (a) We emphasize the functional analytic aspect of our result, which, according to general experience, is also of importance when writing efficient, stable numerical algorithms for solutions of partial differential equations. 
There is room for further research: for example, we have not tried to minimize the numbers $n_{m}$, although this question would probably be relevant from the numerical point of view. Also, we have not made an attempt to consider nonlinear perturbations of the heat equation, although such considerations would no doubt be well motivated in view of $[11,21]$.

(b) By classical arguments, the heat kernel in (1.3) can be expanded as the series

$$
\mathrm{e}^{\frac{1}{4 t}(x-y)^{2}}=\sum_{n \in \mathbb{N}_{0}} \frac{1}{t^{n / 2}} H_{n}(x) y^{n}
$$

where $H_{n}$ are suitably normalized Hermite functions. If $m \in \mathbb{N}$, one can write a given $f$, say, belonging to $L_{w}^{2}(\mathbb{R})$ with $w(x)=\mathrm{e}^{-x^{2} / 2}$, as

$$
f=\sum_{n=1}^{m} f_{n} H_{n}+g
$$

where the coefficients $f_{n}$ are chosen such that $\int_{\mathbb{R}} y^{n} g(y) \mathrm{d} y=0$ for $n=$ $1, \ldots, m$. Then, the solution with initial data $g$ has the decay rate $t^{-(m+1) / 2}$. This known observation yields information resembling our result. However, it does not give a general result on Schauder basis like Theorem 1.1, although it might be used as a starting point for an alternative proof for some special cases of weights, for which the Hermite functions are naturally related. We also mention [6], Appendix A, where analogous results in the form of spectral decompositions are derived for more general equations.

There is an extensive literature dealing with the decay rate of the solution to the Cauchy problem of the heat equation. For example, precise decay rates in the linear case have been considered in [2], although most of the recent research is concentrated on semilinear or other nonlinear generalizations of (1.1)-(1.2). As a slightly random sample, we mention the papers [3-5,8-10,13-15,17,18,23]; see also the monograph [19] for an exposition. We especially mention the papers [1,10-12,20,21], where the asymptotic large time behavior of the semilinear problem is considered by separating the faster decay of terms with vanishing integrals. The paper [11] contains the state of art in this direction and in fact has partially been a source of inspiration for the present work.

As for the contents of our paper, in Sect. 2 we study shrinking Schauder basis and introduce a generalization of the notion, which is necessary to deal with the non-reflexive case $p=1$. The main result is Theorem 2.2 concerning the existence of Schauder basis annihilating given continuous linear functionals. In Sect. 3 we complete the proof of Theorem 1.1 by using the results of Sect. 2 .

We will use the following general notation. By $C, C^{\prime}$, etc., we denote generic positive constants, the exact value of which may change from place to place. The possible dependence, say, on a parameter $p$ is indicated as $C_{p}$. By supp $f$ we denote the support of a function $f$ and by $\operatorname{sp}(A)$ the linear span of a subset $A$ of a vector space. Its closure is denoted by $\overline{\operatorname{sp}(A)}$. We write $\mathbb{N}=\{1,2, \ldots\}, \mathbb{N}_{0}=\{0\} \cup \mathbb{N}$, and $\mathbb{R}^{ \pm}=\{x \in$ $\mathbb{R}: \pm x \geq 0\}$. The characteristic or indicator function of a set $A$ is denoted by $\mathbf{1}_{A}$. 
We use standard notation $L^{p}\left(\mathbb{R}^{N}\right), L^{p}(0,1)$, etc., for unweighted Lebesgue spaces. Moreover, $X^{*}$ stands for the dual of a Banach space $X$, i.e., the space of bounded linear functionals on $X$. The norm of $X^{*}$ is denoted $\|\cdot\|_{X^{*}}$. The identity operator $X \rightarrow X$ is denoted by $\operatorname{id}_{X}$. For a linear operator $T$ between Banach spaces, $\|T\|$ denotes the operator norm.

If $X$ denotes a separable Banach space over the scalar field $\mathbb{K}$ (either $\mathbb{R}$ or $\mathbb{C}$ ), we recall that a sequence $\left(e_{n}\right)_{n=1}^{\infty} \subset X$ is a Schauder basis, or briefly a basis, if every element $f \in X$ can be presented as a convergent sum $f=\sum_{n=1}^{\infty} f_{n} e_{n}$, where the numbers $f_{n} \in \mathbb{K}$ are unique for $f$. For example, in a separable Hilbert space, every orthonormal basis is a Schauder basis. There are many well-known constructions of Schauder bases in classical Banach spaces; among them, the wavelet bases are the most studied in the recent years. We refer to [16,22] for this topic.

\section{On shrinking Schauder bases}

Given a Schauder basis $\left(e_{n}\right)_{n=1}^{\infty}$ of a separable Banach space $X$, we denote for every $n \in \mathbb{N}$ by $P_{n}$ the basis projection

$$
P_{n} f=P_{n}\left(\sum_{k=1}^{\infty} f_{k} e_{k}\right)=\sum_{k=1}^{n} f_{k} e_{k}, \text { where } f=\sum_{k=1}^{\infty} f_{k} e_{k} \in X .
$$

The number $K=\sup _{n}\left\|P_{n}\right\|$ is called the basis constant of $\left(e_{n}\right)_{n=1}^{\infty}$; the supremum defining $K$ is always finite, see [16].

DEFINITION 2.1. Let $x^{*} \in X^{*}$. We say that a Schauder basis $\left(e_{n}\right)_{n=1}^{\infty}$ of $X$ is shrinking with respect to $x^{*}$ if

$$
\lim _{n \rightarrow \infty}\left\|x^{*} \circ\left(\operatorname{id}_{X}-P_{n}\right)\right\|_{X^{*}}=0 .
$$

For a basis $\left(e_{n}\right)_{n=1}^{\infty}$ of $X$, consider the biorthogonal functionals $e_{n}^{*} \in X^{*}$, where $e_{n}^{*}\left(e_{m}\right)=\delta_{m n}$ (Kronecker delta); let $W=\overline{\operatorname{sp}\left\{e_{n}^{*}: n \in \mathbb{N}\right\}} \subset X^{*}$. It is easily seen that $\left(e_{n}^{*}\right)_{n=1}^{\infty}$ is a Schauder basis of $W$ with the basis projections $P_{n}^{*}$, where $P_{n}^{*}\left(x^{*}\right)=x^{*} \circ P_{n}$ for $x^{*} \in X^{*}$. However, we have $W \neq X^{*}$ in general. We obtain that $\left(e_{n}\right)_{n=1}^{\infty}$ is shrinking with respect to $x^{*} \in X^{*}$, if and only if $x^{*} \in W$.

Definition 2.1 extends slightly the classical notion of a shrinking basis, see [16]. A basis $\left(e_{n}\right)_{n=1}^{\infty}$ of $X$ is shrinking, if it is shrinking with respect to all elements in $X^{*}$ in the sense of the preceding definition, i.e., if $W=X^{*}$. In this case $X^{*}$ must be separable. It is well known that every basis of $X$ is shrinking, if $X$ is reflexive. Again, see [16] for more details.

The proof of Theorem 1.1 will be based on the following result.

THEOREM 2.2. Let $x_{m}^{*} \in X^{*}$ for all $m \in \mathbb{N}$, and let $0<\varepsilon<1$. Assume that $\left(\tilde{e}_{n}\right)_{n=1}^{\infty}$ is a Schauder basis of $X$ which is shrinking with respect to all $x_{m}^{*}$. Then, there exists an increasing sequence $\left(n_{m}\right)_{m=1}^{\infty} \subset \mathbb{N}$ and a basis $\left(e_{n}\right)_{n=1}^{\infty}$ of $X$ such that

$$
x_{m}^{*}\left(e_{n}\right)=0 \text { for all } n \geq n_{m} .
$$


If $T: X \rightarrow X$ is the linear change of basis operator with $T \tilde{e}_{n}=e_{n}$ for all $n$, then we have

$$
\left\|\operatorname{id}_{X}-T\right\|<\varepsilon
$$

Obviously, since $0<\varepsilon<1$, condition (2.3) means that $T$ is a bijection and the new basis $\left(e_{n}\right)_{n=1}^{\infty}$ can be considered as perturbation of the given basis $\left(\tilde{e}_{n}\right)_{n=1}^{\infty}$.

For the proof of Theorem 2.2, we need the following elementary and well-known observation.

LEMMA 2.3. Let $\left(g_{n}\right)_{n=1}^{\infty}$ be a basis of the Banach space $Y$ with basis projections $Q_{n}, n \in \mathbb{N}$, and basis constant $K$. Moreover, let $T: Y \rightarrow Y$ be a linear operator with $c:=\left\|\mathrm{id}_{Y}-T\right\|<1$. Then $\left(T g_{n}\right)_{n=1}^{\infty}$ is a basis of $Y$ with basis constant at most $K(1+c) /(1-c)$.

Proof. By the assumption and the Neumann series, $T$ is an isomorphism (linear homeomorphism), and we have $T^{-1}=\sum_{k=0}^{\infty}\left(\operatorname{id}_{Y}-T\right)^{k}$, hence $\left\|T^{-1}\right\| \leq(1-c)^{-1}$. Moreover, $\|T\| \leq 1+\left\|\operatorname{id}_{Y}-T\right\| \leq 1+c$. Hence, $\left(T g_{n}\right)_{n=1}^{\infty}$ is a basis of $Y$ with basis projections $T P_{n} T^{-1}$ and basis constant at most $K\|T\|\left\|T^{-1}\right\| \leq K(1+c) /(1-c)$.

PROPOSITION 2.4. Let $\left(\tilde{g}_{n}\right)_{n=1}^{\infty}$ be a basis of the Banach space $Y$ with basis projections $Q_{n}, n \in \mathbb{N}$, and basis constant $K$. Moreover, let $L, M \in \mathbb{N}$ and assume that $y_{m}^{*} \in Y^{*}, m \in \mathbb{N}$, satisfy

$$
\left.y_{1}^{*}\right|_{\left(\operatorname{id}_{Y}-Q_{L}\right) Y}, \ldots,\left.y_{M}^{*}\right|_{\left(\operatorname{id}_{Y}-Q_{L}\right) Y}=0
$$

and

$$
\lim _{n \rightarrow \infty}\left\|y_{m}^{*} \mid\left(\operatorname{id}_{Y}-Q_{n}\right) Y\right\|=0 \text { for all } m .
$$

Then for any $\delta>0$ there is a basis $\left(g_{n}\right)_{n=1}^{\infty}$ of $Y$ and an index $N>L$ with

$$
\begin{aligned}
& g_{n}=\tilde{g}_{n}, n=1, \ldots, N, \\
& y_{M+1}^{*}\left(g_{n}\right)=0 \text { if } n>N, \quad y_{k}^{*}\left(g_{n}\right)=0 \text { for } k=1, \ldots, M \text { and } n \geq L+1,
\end{aligned}
$$

and

$$
\left\|\operatorname{id}_{Y}-S\right\| \leq \delta
$$

for the linear operator $S: Y \rightarrow Y$ with $S \tilde{g}_{n}=g_{n}$ for all $n \in \mathbb{N}$. The basis constant of $\left(g_{n}\right)_{n=1}^{\infty}$ is at most $K(1+\delta)$.

Proof. If $y_{M+1}^{*} \mid\left(\mathrm{id}_{Y}-Q_{L}\right) Y=0$ then we can take $g_{n}=\tilde{g}_{n}$ for all $n$. Otherwise let $N>L$ be large enough and put

$$
\rho=\frac{\left\|y_{M+1}^{*} \mid\left(\mathrm{id}_{Y}-Q_{N}\right) Y\right\|}{\left\|y_{M+1}^{*} \mid\left(Q_{N}-Q_{L}\right) Y\right\|} .
$$


According to (2.4), we can choose $N$ so large that

$$
(K+1) \rho<\min (\delta, 1) \text { and } K\left(\frac{1+(1+K) \rho}{1-(1+K) \rho}\right)<K(1+\delta) .
$$

In fact $\rho$ can be made arbitrarily small since the denominator in the definition of $\rho$ goes to $\left\|y_{M+1}^{*} \mid\left(\operatorname{id}_{Y}-Q_{L}\right) Y\right\|>0$ if $N$ tends to $\infty$, while the numerator tends to 0 in view of (2.4). We find $x \in\left(Q_{N}-Q_{L}\right) Y$ with $\|x\|=1$ and $y_{M+1}^{*}(x)=\left\|\left.y_{M+1}^{*}\right|_{\left(Q_{N}-Q_{L}\right) Y}\right\|$. (Take into account that $\left(Q_{N}-Q_{L}\right) Y$ is finite dimensional.)

Put $S f=f$ if $f \in Q_{N} Y$ and

$$
S g=g-\frac{y_{M+1}^{*}(g)}{\| y_{M+1}^{*} \mid\left(Q_{N}-Q_{L}\right) Y} x \quad \text { if } g \in\left(\operatorname{id}_{Y}-Q_{N}\right) Y .
$$

Then we have

$$
\|f+g-S(f+g)\|=\|g-S g\| \leq \rho\|g\| \leq \rho(K+1)\|f+g\| \leq \delta\|f+g\| .
$$

Let $g_{n}=S \tilde{g}_{n}$ for all $n$. According to Lemma 2.3 and in view of (2.7), $\left(g_{n}\right)_{n=1}^{\infty}$ is a basis of $Y$ with basis constant smaller than or equal to

$$
K\left(\frac{1+(K+1) \rho}{1-(K+1) \rho}\right) \leq K(1+\delta)
$$

Formula (2.8) yields $y_{M+1}^{*}\left(g_{j}\right)=0$ if $j>N$. Moreover, since $x \in\left(\operatorname{id}_{Y}-Q_{L}\right) Y$, we have $y_{k}^{*}\left(g_{l}\right)=0$ for $k \leq M, l \geq L+1$. Together with (2.9), this proves the proposition.

Conclusion of the proof of Theorem 2.2. Consider $\delta_{n}>0$ such that

$$
\delta_{1}+\sum_{m=2}^{\infty}\left(\prod_{k=1}^{m-1}\left(1+\delta_{k}\right)\right) \delta_{m} \leq \varepsilon, \quad \text { and } K \prod_{n=1}^{\infty}\left(1+\delta_{n}\right) \text { converges. }
$$

Then, we use induction and apply Proposition 2.4 as follows.

We start with the basis $\left(\tilde{e}_{n}\right)_{n=1}^{\infty}=:\left(e_{n}^{(1)}\right)_{n=1}^{\infty}$ and $n_{1}:=0$. If we are in the step $m$, and we already have the indices $n_{k}, k \leq m$, and a basis $\left(e_{n}^{(m)}\right)_{n=1}^{\infty}$ with basis constant at most

$$
K \prod_{k=1}^{m-1}\left(1+\delta_{k}\right)
$$

such that $x_{k}^{*}\left(e_{n}^{(m)}\right)=0$ for all $n \geq n_{k}$ and all $k \leq m$, then we apply Proposition 2.4 with $\tilde{g}_{n}=e_{n}^{(m)}, L=n_{m}, M=m$ and $\delta=\delta_{m}$. This yields an index $N>n_{m}$ and a basis $\left(e_{n}^{(m+1)}\right)_{n=1}^{\infty}$ with basis constant not larger than

$$
K \prod_{k=1}^{m}\left(1+\delta_{k}\right)
$$


such that, in view of (2.5), $e_{n}^{(m+1)}=e_{n}^{(m)}$ for $n \leq N$ and $x^{*}\left(e_{n}^{(m+1)}\right)=0$ for all $n>N$. We obtain

$$
\left\|\mathrm{id}_{X}-S_{m+1}\right\| \leq \delta_{m+1}
$$

for the linear operator $S_{m+1}: X \rightarrow X$ with $S_{m+1} e_{n}^{(m)}=e_{n}^{(m+1)}$ for all $n$. Put $n_{m+1}=N$ and continue the induction.

At the $m$ th step of the process, the first $n_{m}$ elements of the basis remain unchanged so that we end up with a basic sequence (a Schauder basis of its closed linear span) $\left(e_{n}\right)_{n=1}^{\infty}$ with basis constant at most

$$
K \prod_{k=1}^{\infty}\left(1+\delta_{k}\right)
$$

and such that (2.2) holds. In view of (2.6) the linear operator $T: X \rightarrow X$ with $T \tilde{e}_{n}=e_{n}$ for all $n$ satisfies

$$
\begin{aligned}
& \left\|\operatorname{id}_{X}-T\right\| \leq\left\|\operatorname{id}_{X}-S_{1}+S_{1}-S_{2} S_{1}+S_{2} S_{1}-S_{3} S_{2} S_{1}+\cdots\right\| \\
& \quad \leq\left\|\operatorname{id}_{X}-S_{1}\right\|+\sum_{m=2}^{\infty}\left\|\operatorname{id}_{X}-S_{m}\right\| \prod_{k=1}^{m-1}\left\|S_{k}\right\| \\
& \quad \leq \delta_{1}+\sum_{m=2}^{\infty}\left(\prod_{k=1}^{m-1}\left(1+\delta_{k}\right)\right) \delta_{m} \leq \varepsilon .
\end{aligned}
$$

If we choose $\varepsilon<1$, then $T$ is surjective and $\left(e_{n}\right)_{n=1}^{\infty}$ is a basis of $X$ with the required properties.

\section{Proof of Theorem 1.1}

We start the proof of Theorem 1.1 by some preparations. Given a measurable function $g$ on $\mathbb{R}^{N}$, we denote by $\Phi_{g}$ the functional

$$
\Phi_{g}(f)=\int_{\mathbb{R}^{N}} g(y) f(y) \mathrm{d} y,
$$

which is defined for measurable functions $f$ on $\mathbb{R}^{N}$ such that the integral converges. Let us first show the following result.

THEOREM 3.1. Let $1 \leq p<\infty$ and let for all $m \in \mathbb{N}$ the functions $h_{m}: \mathbb{R}^{N} \rightarrow \mathbb{R}$ be measurable such that, if $p>1$,

$$
\int_{\mathbb{R}^{N}}\left(\frac{\left|h_{m}(y)\right|^{p}}{w(y)}\right)^{1 /(p-1)} \mathrm{d} y<\infty,
$$

or, if $p=1$,

all $h_{m} / w$ are continuous and can be continuously

extended to $[-\infty, \infty]^{N}$. 
Then, every $\Phi_{h_{m}}$ defines a bounded linear functional on $L_{w}^{p}\left(\mathbb{R}^{N}\right)$, and there are a Schauder basis $\left(e_{n}\right)_{n=1}^{\infty}$ of $L_{w}^{p}\left(\mathbb{R}^{N}\right)$ and indices $n_{1}<n_{2}<\cdots$ such that

$$
\Phi_{h_{m}}\left(e_{n}\right):=\int_{\mathbb{R}^{N}} h_{m}(y) e_{n}(y) \mathrm{d} y=0 \text { for all } n \geq n_{m}
$$

Moreover, if $p>1$, a basis $\left(\tilde{e}_{n}\right)_{n=1}^{\infty} \subset X$ is given and $\varepsilon>0$ is arbitrary, then the above mentioned basis $\left(e_{n}\right)_{n=1}^{\infty}$ can be chosen such that

$$
\left\|\operatorname{id}_{X}-T\right\|<\varepsilon
$$

where $T: X \rightarrow X$ is the linear operator defined by with $T \tilde{e}_{n}=e_{n}$ for all $n$.

Proof. If $p>1$, then (3.1) implies, for every $f \in L_{w}^{p}\left(\mathbb{R}^{N}\right)$,

$$
\begin{aligned}
& \left|\int_{\mathbb{R}^{N}} h_{m}(y) f(y) \mathrm{d} y\right| \leq \int_{\mathbb{R}^{N}} \frac{\left|h_{m}(y)\right|}{w(y)^{1 / p}}|f(y)| w(y)^{1 / p} \mathrm{~d} y \\
& \quad \leq\left(\int_{\mathbb{R}^{N}}\left(\frac{\left|h_{m}(y)^{p}\right|}{w(y)}\right)^{1 /(p-1)} \mathrm{d} y\right)^{(p-1) / p}\left(\int_{\mathbb{R}^{N}}|f(y)|^{p} w(y) \mathrm{d} y\right)^{1 / p}
\end{aligned}
$$

in view of the Hölder inequality. This means that $\Phi_{h_{m}}$ is a bounded linear functional on $L_{w}^{p}\left(\mathbb{R}^{N}\right)$. Since $L_{w}^{p}\left(\mathbb{R}^{N}\right)$ is reflexive, the theorem follows from Theorem 2.2.

Let us deal with the case $p=1$. We consider the Haar system $\left(e_{n}\right)_{n=1}^{\infty}$ in $L^{1}(0,1)$, where $e_{1} \equiv 1$ and

$$
e_{2^{k}+j}(t)=\left\{\begin{aligned}
1, & \text { if } t \in\left[(2 j-2) 2^{-k-1},(2 j-1) 2^{-k-1}\right], \\
-1, & \text { if } t \in\left[(2 j-1) 2^{-k-1},(2 j) 2^{-k-1}\right], \\
0, & \text { otherwise, }
\end{aligned}\right.
$$

for $k=0,1,2, \ldots$ and $j=1, \ldots, 2^{k}$. It is well known that the Haar system is a Schauder basis for $L^{1}(0,1)$ with basis constant 1 . Put

$$
A_{2^{k}+j-1}=\left[\frac{j-1}{2^{k}}, \frac{j}{2^{k}}\right] .
$$

Then, we have $\mathbf{1}_{A_{1}}=e_{1}, \mathbf{1}_{A_{2}}=\left(e_{1}+e_{2}\right) / 2$ and $\mathbf{1}_{A_{3}}=\left(e_{1}-e_{2}\right) / 2$. By induction we see that any element $\mathbf{1}_{A_{m}}$ is a linear combination of the Haar functions $e_{n}$.

It is well known that there is a linear order on the indices $\alpha=\left(n_{1}, \ldots, n_{N}\right)$ such that the functions $\tilde{u}_{\alpha}$ with

$$
\tilde{u}_{\alpha}\left(s_{1}, \ldots, s_{N}\right)=e_{n_{1}}\left(s_{1}\right) \cdot \ldots \cdot e_{n_{N}}\left(s_{N}\right), \quad\left(s_{1}, \ldots, s_{N}\right) \in[0,1]^{N}
$$

form a Schauder basis of $L^{1}\left([0,1]^{N}\right)$ whose biorthogonal functionals are, up to constant factors, the functionals $\Psi_{\tilde{u}_{\alpha}}$ with

$$
\Psi_{\tilde{u}_{\alpha}}(f)=\int_{[0,1]^{N}} \tilde{u}_{\alpha}(s) f(s) d s
$$


and $\left\|\Psi_{\tilde{u}_{\alpha}}\right\|=\left\|\tilde{u}_{\alpha}\right\|_{\infty}$, see [7]. We obtain that all functions of the form $\mathbf{1}_{A_{k_{1}} \times \cdots \times A_{k_{N}}}$ are elements of the linear span of the basis elements $\tilde{u}_{\alpha}$.

Now define $\gamma:] 0,1\left[{ }^{N} \rightarrow \mathbb{R}^{N}\right.$ by

$$
\gamma(s)=\left(\tan \left(\pi\left(s_{1}-2^{-1}\right)\right), \ldots, \tan \left(\pi\left(s_{N}-2^{-1}\right)\right)\right), \quad \text { for } s=\left(s_{1}, \ldots, s_{N}\right)
$$

and

$$
(S f)(s)=f(\gamma(s)) w(\gamma(s)) \prod_{k=1}^{N} \frac{\pi}{\cos ^{2}\left(\pi\left(s_{k}-2^{-1}\right)\right)} \quad \text { for } f \in L_{w}^{1}\left(\mathbb{R}^{N}\right) .
$$

Then, $S$ is an isometric isomorphism between $L_{w}^{1}\left(\mathbb{R}^{N}\right)$ and $L^{1}\left([0,1]^{N}\right)$. Since

$$
\int_{\mathbb{R}^{N}} h_{m}(y) f(y) \mathrm{d} y=\int_{[0,1]^{N}} \frac{h_{m}(\gamma(s))}{w(\gamma(s))}(S f)(s) d s,
$$

we obtain $\Phi_{h_{m}}(f)=\Psi_{h_{m} \circ \gamma / w \circ \gamma}(S f)$. In view of the assumptions (3.2), the function $h_{m} \circ \gamma / w \circ \gamma$ can be continuously extended from $] 0,1{ }^{N}$ to $[0,1]^{N}$ and hence is uniformly continuous there. Therefore, we find, for every $\varepsilon>0$, a linear combination $g$ of the functions $\mathbf{1}_{A_{k_{1}} \times \cdots \times A_{k_{N}}}$ with $\left\|g-h_{m} \circ \gamma / w \circ \gamma\right\|_{\infty} \leq \varepsilon$. Hence $\Psi_{h_{m} \circ \gamma / w \circ \gamma}$ are elements of the norm closure of the linear span of the biorthogonal functionals of $\left(\tilde{u}_{\alpha}\right)$. This means that the basis $\left(\tilde{u}_{\alpha}\right)$ is shrinking for the functionals $\Psi_{h_{m} \circ \gamma / w \circ \gamma}$. Then, Theorem 2.2 yields a basis $\left(u_{n}\right)_{n=1}^{\infty}$ of $L^{1}\left([0,1]^{N}\right)$ and indices $n_{1}<n_{2}<\cdots$ such that $\Psi_{h_{m} \circ \gamma / w \circ \gamma}\left(u_{n}\right)=0$ if $n \geq n_{m}$. Put $e_{n}=S^{-1} u_{n}$. Hence, $\left(e_{n}\right)_{n=1}^{\infty}$ is a basis of $L_{w}^{1}\left(\mathbb{R}^{N}\right)$ which satisfies the assertion of the theorem in view of (3.5).

We now return to the proof of Theorem 1.1. Fix $x \in \mathbb{R}^{N}, t>0$ and put

$$
g(y)=\exp \left(-y^{2}\right), \quad g_{x}(y)=g((x-y) / \sqrt{4 t}), \quad y \in \mathbb{R}^{N} .
$$

For any $\alpha \in \mathbb{N}_{0}^{N}$, we have

$$
D^{\alpha} g_{x}(y)=\frac{(-1)^{|\alpha|}}{(4 t)^{|\alpha| / 2}}\left(D^{\alpha} g\right)((x-y) / \sqrt{4 t}) .
$$

Let us denote by $m(\alpha) \in \mathbb{N}_{0}$ an ordering all multi-indices $\alpha$ such that in particular $m(\alpha)<m(\beta)$ for all $\alpha, \beta$ with $|\alpha|<|\beta|$. Then, we define for every $m \in \mathbb{N}_{0}$ the functions $h_{m}$,

$$
h_{m}(y)=y^{\alpha}, \quad y \in \mathbb{R}^{N}
$$

where $\alpha$ is such that $m=m(\alpha)$.

It follows easily from the choice of the weight in (1.4), that the functions $h_{m}$ satisfy the assumptions of Theorem 3.1 so that $\Phi_{h_{m}}$ are bounded functionals on $L_{w}^{p}\left(\mathbb{R}^{N}\right)$ and we find a Schauder basis $\left(e_{n}\right)_{n=1}^{\infty}$ of $L_{w}^{p}\left(\mathbb{R}^{N}\right)$ and indices $v_{1}<v_{2}<\cdots$ such that

$$
\Phi_{h_{m}}\left(e_{n}\right)=0 \text { for all } n \geq v_{m} \text {. }
$$


We define the numbers $n_{m}$ appearing in the theorem, as follows: if $m(\alpha)$ is the largest number under the condition $|\alpha|<m$, then we set

$$
n_{m}:=v_{m(\alpha)}
$$

Thus, the choice of the numbers $m(\alpha)$ and (3.9) implies that

$$
\Phi_{h_{m(\alpha)}}\left(e_{n}\right)=0 \text { for all } n \geq n_{m} \text {, all } \alpha \text { with }|\alpha|<m \text {. }
$$

The statement about the change of basis operator in Theorem 1.1 follows from (3.3). There remains to show the fast decay property (1.6). Let us fix an arbitrary $\tilde{m} \in \mathbb{N}$. The multidimensional Taylor formula yields a function $\bar{y}(y)$ such that

$$
g_{x}(y)=\sum_{|\alpha|<\tilde{m}} \frac{D^{\alpha} g_{x}(0)}{\alpha !} y^{\alpha}+\sum_{|\alpha|=\widetilde{m}} \frac{D^{\alpha} g_{x}(\bar{y}(y))}{\alpha !} y^{\alpha} .
$$

For $\alpha$ with $|\alpha|=\tilde{m}$, we obtain constants $c_{\tilde{m}}$ (in particular independent of $t$ ), such that

$$
\sup _{x, y}\left|\frac{D^{\alpha} g_{x}(\bar{y}(y))}{\alpha !}\right| \leq \frac{c_{\tilde{m}}}{t^{\widetilde{m} / 2}},
$$

since $g$ is the Gaussian function. Now let $f=\sum_{n \geq n_{\tilde{m}}} f_{n} e_{n} \in L_{w}^{p}\left(\mathbb{R}^{N}\right)$. We get by (3.12)

$$
\begin{aligned}
\left|\mathrm{e}^{t A} f(x)\right|= & \frac{1}{(2 \pi t)^{N / 2}}\left|\int_{\mathbb{R}^{N}} g_{x}(y) f(y) \mathrm{d} y\right| \\
= & \frac{1}{(2 \pi t)^{N / 2}} \mid \sum_{|\alpha|<\tilde{m}} \frac{D^{\alpha} g_{x}(0)}{\alpha !} \Phi_{h_{m(\alpha)}}\left(\sum_{n \geq n_{\tilde{m}}} f_{n} e_{n}\right) \\
& +\sum_{|\alpha|=\tilde{m}} \int_{\mathbb{R}^{N}} \frac{D^{\alpha} g_{x}(\bar{y}(y))}{\alpha !} y^{\alpha} f(y) \mathrm{d} y \mid
\end{aligned}
$$

Here, due to (3.11), only the last line is nonzero, and if $p>1$, it can be bounded using (3.13) by

$$
\begin{aligned}
& \sum_{|\alpha|=\tilde{m}} \frac{C}{t^{(N+\widetilde{m}) / 2}} \int_{\mathbb{R}^{N}}\left|\frac{D^{\alpha} g_{x}(\bar{y}(y))}{\alpha !} y^{\alpha} f(y)\right| \mathrm{d} y \\
& \leq \sum_{|\alpha|=\tilde{m}} \frac{C c_{\tilde{m}}}{t^{(N+\widetilde{m}) / 2}} \int_{\mathbb{R}^{N}}\left|y^{\alpha} f(y)\right| \mathrm{d} y \\
& \leq \sum_{|\alpha|=\tilde{m}} \frac{C c_{\tilde{m}}}{t^{(N+\widetilde{m}) / 2}}\left(\int_{\mathbb{R}^{N}}\left|y^{\alpha}\right|^{q} w(y)^{-q / p} \mathrm{~d} y\right)^{1 / q}\left(\int_{\mathbb{R}^{N}} w(y)|f(y)|^{p} \mathrm{~d} y\right)^{1 / p} \\
& \leq \frac{C_{\widetilde{m}}^{\prime}}{t^{(N+\widetilde{m}) / 2}}\|f\|_{p, w}
\end{aligned}
$$

where $C_{\widetilde{m}}^{\prime}$ and $C$ are constants, $q$ is the dual exponent of $p$ and the last but one integral converges by (1.4). The modification for the case $p=1, q=\infty$ is obvious. This yields (1.6) since $\tilde{m}$ can be chosen arbitrarily large. 


\section{Acknowledgements}

Open access funding provided by University of Helsinki including Helsinki University Central Hospital. The authors would like to thank Thierry Gallay (Grenoble) for discussions which helped in the final formulation of our results. The research of Bonet was partially supported by the projects MTM2016-76647-P and GV Prometeo 2017/102. The research of Taskinen was partially supported by the research grant from the Faculty of Science of the University of Helsinki.

Open Access. This article is distributed under the terms of the Creative Commons Attribution 4.0 International License (http://creativecommons.org/licenses/by/4.0/), which permits unrestricted use, distribution, and reproduction in any medium, provided you give appropriate credit to the original author(s) and the source, provide a link to the Creative Commons license, and indicate if changes were made.

Publisher's Note Springer Nature remains neutral with regard to jurisdictional claims in published maps and institutional affiliations.

\section{REFERENCES}

[1] Bricmont, J., Kupiainen, A., and Lin, G., Renormalization group and asymptotics of solutions of nonlinear parabolic equations. Comm. Pure Appl. Math. 47 (1994), 839-922.

[2] Cazenave, T., Dickstein, F., Weissler, F., A solution of the heat equation with a continuum of decay rates. Elliptic and parabolic problems, 135-138, Prog. Nonlinear Differential Equations Appl., 63, Birkhäuser, Basel, 2005. 35K05 (35B40)

[3] Escobedo, M., Kavian, O., Variational problems related to self-similar solutions of the heat equation. Nonlin. Anal. 11 (1987), 1103-1133.

[4] Fila, M., Winkler, M., and Yanagida, E., Convergence rate for a parabolic equation with supercritical nonlinearity. J. Dyn. Diff. Eq 17 (2005), 249-269.

[5] Fujita, H., On the blowing up of the solutions of the Cauchy problem for $u_{t}=\Delta u+u^{1+\alpha}$. J. Fac. Sci. Univ. Tokyo Sect IA 13 (1966), 109-124.

[6] Gallay, Th., Wayne, C.E., Invariant Manifolds and the Long-Time Asymptotics of the Navier-Stokes and Vorticity Equations on $\mathbf{R}^{2}$. Arch. Rational Mech. Anal. 163 (2002), 209-258.

[7] Gelbaum, B.R., Gil de Lamadrid, J., Bases of tensor products of Banach spaces. Pacific J. Math. 11 (1961), 1281-1286.

[8] Giga, Y., A bound for global solutions of semilinear heat equations. Comm. Math. Phys. 103 (1986), 415-421.

[9] Gmira, A., and Veron, L.: Large time behavior of the solutions of a semilinear parabolic equation in $\mathbf{R}^{N}$. J. Diff. Eq. 53 (1984), 258-276.

[10] Ishige, K., and Kawakami, T., Asymptotic behavior of solutions for some semilinear heat equations in $\mathbb{R}^{N}$. Commun. Pure Appl. Anal. 8 (2009), no. 4, 1351-1371.

[11] Ishige, K., Kawakami, T., Refined asymptotic profiles for a semilinear heat equation. Math. Ann. 353 (2012), no. 1, 161-192.

[12] Ishige, K., and Kawakami, T., Asymptotic expansions of solutions of the Cauchy problem for nonlinear parabolic equations. J. Anal. Math. 121 (2013), 317-351.

[13] Kavian, O., Remarks on the large time behaviour of a nonlinear diffusion equation. Ann. Inst. H. Poincaré, Analyse Non Linéaire 4 (1987), 423-452.

[14] Kawanago, T., Asymptotic behavior of solutions of a semilinear heat equation with subcritical nonlinearity. Ann. Inst. H. Poincaré, Analyse Non Linéaire 13 (1996), 1-15.

[15] Kobayashi, K., Sirao, T., and Tanaka, H., On the growing up problem for semi-linear heat equations. J.Math. Soc. Japan 29 (1977), 407-424.

[16] Lindenstrauss,J., Tzafriri, J., Classical Banach spaces I, Springer, Berlin, 1977. 
[17] Poláčik, P,. and Quittner, P., Asymptotic behavior of threshold and sub-threshold solutions of a semilinear heat equation. Asymptot. Anal. 57, 3-4 (2008), 125-141.

[18] Quittner, P., The decay of global solutions of a semilinear heat equation. Discr. Cont. Dynamical Systems. 21,1(2008). 307-318.

[19] Quittner, P., Souplet, Ph., Superlinear parabolic problems. Blow-up, global existence and steady states. Birkhäuser advanced texts, Basel, 2007.

[20] Taskinen, J., Asymptotical behaviour of a class of semilinear diffusion equations. J. Evol. Eq. 7 (2007), 429-447.

[21] Taskinen, J., Long-time asymptotics of sub-threshold solutions of a semilinear Cauchy problem. Diff. Eq. and Applications 3 (2) (2011), 279-297.

[22] Wojtaszyck, P., Banach spaces for analysts. Cambridge Studies in Advanced Mathematics, vol 25. Cambridge University Press, Cambridge, 1991.

[23] Zhao, H., Large time decay estimates of solutions of nonlinear parabolic equations. Discrete and Continuous Dynamical Systems 8,1 (2002), 69-144.

\author{
José Bonet \\ Instituto Universitario de Matemática Pura y Aplicada IUMPA \\ Universitat Politècnica de València \\ 46071 Valencia \\ Spain \\ E-mail: jbonet@mat.upv.es \\ Wolfgang Lusky \\ Institut für Mathematik \\ Universität Paderborn \\ 33098 Paderborn \\ Germany \\ E-mail: lusky@uni-paderborn.de \\ Jari Taskinen \\ Department of Mathematics and Statistics \\ University of Helsinki \\ P.O. Box 68 \\ o0014 Helsinki \\ Finland \\ E-mail: jari.taskinen@helsinki.fi
}

